\title{
Phosphoproteomics of Cold Stress-Responsive Mechanisms in Rhododendron Chrysanthum
}

\author{
yunbo liu \\ Jilin Normal University \\ Ziyao Zhang \\ Jilin Normal University \\ Hang Fan \\ Jilin Normal University \\ Yun Tan \\ Jilin Normal University \\ Xiaofu Zhou \\ Jilin Normal University \\ Hongwei Xu ( $\square$ xuhongwei@jInu.edu.cn ) \\ Jilin Normal University
}

Research

Keywords: Rhododendron chrysanthum Pall., Phosphoproteomics, cold stress,

Posted Date: June 9th, 2021

DOl: https://doi.org/10.21203/rs.3.rs-585853/v1

License: (c) (i) This work is licensed under a Creative Commons Attribution 4.0 International License.

Read Full License 


\section{Abstract}

Background: As an alpine plants $₫$ Rhododendron chrysanthum ( $R$. chrysanthum) has evolved cold resistance mechanisms and become a valuable plant resource with the responsive mechanism of cold stress.

Results: In my study, we adopt the phosphoproteomic and proteomic analysis combining with physiological measurement to illustrate the responsive mechanism of $R$. chrysanthum seedling under cold $\left(4^{\circ} \mathrm{C}\right)$ stress. After chilling for $12 \mathrm{~h}, 350$ significantly changed proteins and 274 significantly changed phosphoproteins were detected. COG analysis showed that significantly changed proteins and phosphoproteins were mainly involved in signal transduction and energy production and conversion under cold stress. The results indicated photosynthesis was inhibited under cold stress, but cold induced calcium-mediated signaling, reactive oxygen species (ROS) homeostasis and other transcription regulation factors could protect plants from destruction caused by cold stress.

Conclusions: These data constitute a cold stress-responsive metabolic atlas in R. chrysanthum, which will springboard further investigations into the complex molecular mechanisms of plant cold adaptation.

\section{Background}

As one of the major environmental factors, cold affects the plant growth and development broadly [1]. Cold stress directly leads to membrane rigidification and protein denaturation, but also indirectly reduces the activities of enzymes [2], inhibition of protein synthesis and degradation, as well as considerable membrane damage [3]. In order to reduce limit cold-induced damage, plants especially alpine plants have evolved multiple stress-tolerant strategies, which contribute to protect plants from destruction caused by cold stress [4]. Photosynthetic process of plants is inevitably changed in response to cold stress, especially PS囚. The PSII photoinhibition appeared in order to protect PSI at cold stress [5]. Cold stress induced the changes of a variety of protein kinases and transcription factors in plants [6]. According to reports, calcium plays a vital role in cold stress signaling [7]. Various abiotic stresses result in excessive accumulation of reactive oxygen species (ROS) in plants, and the effective antioxidant enzymes such as catalase (CAT), Superoxide dismutase (SOD) and peroxidase (POD) could eliminate excessive ROS [8].

As the major post-translational modification, protein phosphorylation plays a crucial roles in providing stress signal transduction and the regulation of diverse biological functions in plants [9]. Therefore, protein phosphorylation events means a lot in designing strategies preventing plants from cold and other abiotic stresses, and it should be included when studying stress-induced related signal pathways [10]. Phosphoproteomic responses to cold stress have been investigated in different parts from different plants, including the leaves of Arabidopsis [9], tomato [11], Paper Mulberry [12],Tobacco [13].

Rhododendron chrysanthum Pall. ( $R$. chrysanthum), an alpine plant, which is a valuable germplasm resource in the world with important ornamental and medicinal value $R$. chrysanthum only grows at altitudes around $1700 \mathrm{~m}$ at the Changbai Mountain in Jilin Province in China. In the long-term adaptive 
evolution, $R$. chrysanthum has evolved sophisticated mechanisms to respond to cold stress and protect itself from freezing injury [14].

Previous studies on the cold stress mechanism of cash crops and tropical plants are more thorough. Nevertheless, only few studies focused on molecular mechanism of cold response in alpine plants. In this study, quantitative proteomics and phosphoproteomics combining with traditional physiological analyses were employed to explore the cold response and defense mechanism in rhododendron chrysanthum leaves at phosphorylation level. These results have improved our understanding how is rhododendron chrysanthum respond to cold stress and further unveil the specific phosphorylated proteins involved in potential pathways in rhododendron chrysanthum under cold stress.

\section{Results}

Changes of Photosynthetic Characteristics in Response to Cold Stress

Photosynthesis of rhododendron chrysanthum is very sensitive to cold stress. The Fo and Fm significantly decreased under cold stress condition (Fig. 1A, B). Fv/Fo also decreased in response to the cold stress (Fig. 1E). In addition, QP and NPQ were considered as two indexes of utility rate of luminous energy. The decrease of $\mathrm{qP}$ and NPQ reflects the decrease of light energy utilization under cold stress (Fig. 1G, H). The parameters Fv/Fm and Fv'/Fm' represent the maximal and effective quantum yield of $\mathrm{PS} \otimes$ photochemistry, respectively. Fv/Fm decreased during the treatment (Fig. 1C, D), while Fv'/Fm' was significantly reduced under cold stress. However, there was no significant change in ETR under cold stress (Fig. 1F).

Effects of cold Stress on Antioxidant Enzyme Systems

The $\mathrm{H} 2 \mathrm{O} 2$ content significantly increased under cold stress condition (Fig. 2A). The activities of the antioxidant enzymes were also affected by cold stress. CAT activity significantly increased with the cold stress.

Cold Stress-Responsive Proteome in rhododendron chrysanthum Leaves

The cold stress-responsive proteins abundance pattern in rhododendron chrysanthum was analyzed using iTRAQ-based quantitative proteomic approach. In total, 5,192 protein species in leaves were identified in at least three independent biological replicates, 350 of which were defined as cold stressresponsive proteins using a threshold of significance of $p<0.05$, and with a fold change $>1.2$ in protein abundance. Among them, 173 protein species increased and 177 decreased under the cold stress conditions (Fig. 3A). The cold stress-responsive proteins were classified into 20 functional categories (Fig. 3B). The cold stress-responsive mainly functioned in Energy production and conversion, Signal transduction mechanisms. The majority of the cold stress-increased proteins in Signal transduction indicated that active signaling and metabolic networks have been initiated in rhododendron chrysanthum leaves to cope with the cold stress. 
In rhododendron chrysanthum leaves, 2872 phosphopeptides representing 2508 phosphoproteins were identifified using a TiO2 enrichment-based proteomics approach. Among them, 274 cold stressresponsive phosphopeptides representing 193 phosphoproteins (belonging to 12 functional categories) were detected with more than 1.2 -fold changes $(p<0.05)$ of phosphorylation level, including 22 cold stress-increased and 252 cold stress-decreased phosphopeptides (Figs. 3A). A total of 274 cold stressresponsive phosphopeptides were classified into 8 functional categories. There were 252 phosphopeptides' phosphorylation levels decreased under cold stress condition. The corresponding proteins were mainly involved in Energy production and conversion, Signal transduction mechanisms.

Three-Dimensional Structure Modeling of Heat Stress-Responsive Phosphoproteins

In order to better understand the biochemical functions of the protein phosphorylation during the cold stress response, we predicted the molecular structure of cold stress-responsive phosphoproteins. In total, 6 statistically acceptable homology models were built through the SWISS-MODEL and their phosphorylation sites were located within the three-dimensional structure models (Fig. 4). The phosphorylation levels of Photosystem II protein D1 and light-harvesting complex II chlorophyll a/b binding protein 1(LHCB1) increased under heat stress and the phosphorylation levels of phosphoglycerate kinase, fructose-bisphosphate aldolase, transketolase and mitogen-activated protein kinases decreased under heat stress. The cold stress-increased phosphorylation site of D1 protein occurred on Ser232, which was located in the PEST-like region [15] (Fig. 4A). However, the phosphorylation sites of other proteins were not located in their functional domain.

\section{Discussion}

Photosynthesis Is Inhibited Under Cold Stress

In this study, photosynthetic parameters [i.e., Fm, Fo, Fv/Fo and Fv'/Fm'] of rhododendron chrysanthum were obviously reduced with the cold treatment (Fig. 1), indicating the damage of the photosystem in rhododendron chrysanthum. However, there was no significant change in ETR and Fv/Fm, which might mean that there are some strategies in rhododendron chrysanthum to alleviating PSIl damage.

Phosphorylation of PS II centers increases the stability of PS II complexes and concomitantly improves plant tolerance to cold stress [16]. In this study, as the center of PS II, D1 protein was significantly phosphorylated under cold stress, which might result from the accumulation of ROS. The degradation of the D1 reduced following phosphorylation of protein [17]. In addition, we observed that the phosphorylation level of Ihcb4(CP 29) and Ihcb1(LHCB 2) dramatically increased (Fig. 5), which might lead a decreased in NPQ. Similar results had been illuminated at Picea abies in cold stress [18]. These results suggest that these proteins plays a role in sustained thermal dissipation [19].The decrease of phosphorylation level of PsbR indicated that PSIl oxygen evolving complex(OEC) might be damaged under the cold stress [20]. However, NAD(P)+-dependent aldehyde dehydrogenases (ALDH) involved in 
oxidation of reactive aldehydes decreased in rhododendron chrysanthum under cold stress [21]. The cold stress also decreased the phosphorylation levels of Calvin cycle enzymes e.g., fructose-bisphosphate aldolase and transketolase (Fig. 5), which might lead to the decrease of Calvin cycle activity under cold stress [22].

Diverse ROS Scavenging Pathways Are Employed for cold Stress Responses

In rhododendron chrysanthum leaves, cold stress caused the accumulation of $\mathrm{H}_{2} \mathrm{O}_{2}$ (Fig. 2A). In respond to oxidative damage caused by the cold stress, rhododendron chrysanthum has developed enzymatic detoxification systems to counteract ROS toxicity. ROS could also induce some secondary messengers like MAPK, and impact on cold signaling [6]. As we know, in response to oxidative stress, plant tissues would increase the activity of SOD to reduce the ROS level and generate $\mathrm{H}_{2} \mathrm{O}_{2}$ [8]. The SOD was significantly enhanced under cold stress (Fig. 6), which is in agreement with the cold stress-increased SOD activity in rice [23] and rapeseed [24]. In addition, cold stress significantly enhanced the CAT activity(Fig. 2B), which is supposed to break down $\mathrm{H}_{2} \mathrm{O}_{2}$ into water and oxygen [25].CAT is also distinguished from many other peroxide-metabolizing enzymes due to its high specificity for $\mathrm{H}_{2} \mathrm{O}_{2}$ [26]. Enzymes capable of eliminating $\mathrm{H}_{2} \mathrm{O}_{2}$ include Prx. Phosphorylation of Prx reduced the peroxidase activity of this protein [27].In this study, cold stress decrease of phosphorylation level of Prx, might suggest that cold stress enhanced activity of Prx. However, The POD pathway was inhibited in rhododendron chrysanthum leaves as evidenced by the cold stress-decreased abundances of all the isoenzymes of POD. Similar result had been illuminated at rice under heat stress [28]. that NDPK is associated with $\mathrm{H}_{2} \mathrm{O}_{2}$-mediated mitogen-activated protein kinase signaling in plants [29]. In this study, the phosphorylation levels of NDPH and MAP3K are similar.

Diverse Calcium-Mediated Signaling Pathways Are Induced in the Cold-Tolerant rhododendron chrysanthum

It is becoming clear that $\mathrm{Ca} 2+$ signaling plays a crucial role in conferring cold tolerance in plants [7]. In addition, the calcium would bind to the calmodulin [30] to phosphorylate calcium/CaM-binding protein (CaM-BP) to regulate gene expression [31]. In this study, the cold stress-phosphorylated CaM-binding protein implied their possible involvement in calcium/CaM signaling events (Fig. 8). The cold increased calnexin and calreticulin may promote the intracellular calcium homeostasis and signaling in rhododendron chrysanthum leaves (Fig. 8) [32]. It was reported that serine/threonine-protein kinase BLUS1 functioned as primary regulator of stomatal control to enhance photosynthetic $\mathrm{CO} 2$ assimilation [33].In this study, cold stress decreased the phosphorylation levels of serine/threonine-protein kinase (Fig. 8), which might lead to stomatal closure in rhododendron chrysanthum leaves. Similar result had been illuminated at Jatropha Curcas Seedling under cold stress [4]. All these results indicate calciummediated signaling pathways is induced and lead to cold-responsive gene expression in the cold stress.

\section{Conclusion}


As an alpine plant, $R$. chrysanthum is excellent material for plants cold respond research. Although, our physiological results indicated that the photosynthesis of $R$. chrysanthum was inhibited,the quantitative proteomics and phosphoproteomics results implied that diverse ROS scavenging pathways and calciummediated signaling was triggered to alleviate damage under cold stress. All these results provide valuable information about the molecular mechanism of the cold tolerance of Rhododendron chrysanthum Pall.

\section{Materials And Methods}

\section{Plant materials and experimental design}

Wild Rhododendron chrysanthum tissue seedlings were exposed to $4{ }^{\circ} \mathrm{C}$ for $12 \mathrm{~h}$ were used as the experimental group (EG). Wild Rhododendron chrysanthum tissue seedlings were exposed to normal atmospheric temperature for $12 \mathrm{~h}$ were used as the control group (CG). Both $\mathrm{EG}$ and $\mathrm{CG}$ The leaves excised from six -month-old plants of the EG and the CG were immediately used for protein extraction. To ensure adequately coverage, three biological replicates of each group (i.e., six plants) were collected.

\section{Chlorophyll fluorescence measurement}

Chlorophyll fluorescence induction parameters of EG and CG leaves were carried out with the Maxiversion of the Imaging-PAM (Walz, Germany). Before measurement, the plants were kept in darkness for 30 min to allow all reaction centers to open. Then, the fourth leaf from the top of each plant was detached and clamped onto the holder. The minimal fluorescence (Fo) of dark-adapted leaves was recorded during the weak measuring pulses of $0.5 \mu \mathrm{mol} \mathrm{m} \mathrm{m}^{-2} \mathrm{~s}^{-1}$ and the maximal quantum yield of PSII photochemistry $(\mathrm{Fm})$ was obtained upon application of a $0.5 \mathrm{~s}$ saturation light pulse of $2,800 \mu \mathrm{mol} \mathrm{m}^{-2}$ $\mathrm{s}^{-1}$. The intensity of actinic light setting used in all trials was $230 \mu \mathrm{mol} \mathrm{m} \mathrm{m}^{-2} \mathrm{~s}^{-1}$. The maximal quantum yield of PSII photochemistry $\left(F_{v} / F_{m}\right)$, effective quantum yield of PSIl photochemistry $\left(F_{v}{ }^{\prime} / F_{m}{ }^{\prime}\right)$, nonphotochemical quenching (NPQ), photochemical quenching (qP), and electron transport rate (ETR) were calculated using ImagingWin version 2.39 software (Walz). Statistical analysis was performed by using SAS 9.4. All data are represented as the means \pm SD with three biological independent replications.

\section{H2O2 Content and antioxidant enzyme activity assays}

The activity of catalase (CAT) and the content of $\mathrm{H}_{2} \mathrm{O}_{2}$ within leaves were determined by Plant CAT ELISA kit and Plant $\mathrm{H}_{2} \mathrm{O}_{2}$ ELISA kit (Shanghai Enzyme Biotechnology Co., Ltd., China) according to the manufacturers instructions.

\section{Protein extraction}

Two grams of leaves were ground in liquid nitrogen and mixed with lysis buffer ( $8 \mathrm{M}$ urea, $2 \mathrm{mM}$ EDTA, $10 \mathrm{mM}$ DTT and $1 \%$ Protease Inhibitor Cocktail). The mixture was sonicated three times on ice using a high-intensity ultrasonic processor (Scientz, China). The remaining debris were removed by centrifugation at $20,000 \times \mathrm{g}$ at $4^{\circ} \mathrm{C}$ for $10 \mathrm{~min}$. The protein in the supernatant was precipitated with cold $15 \%$ TCA at $-20^{\circ} \mathrm{C}$ for $4 \mathrm{~h}$. After centrifugation at $4^{\circ} \mathrm{C}$ for 3 min, the remaining precipitates were washed with cold 
acetone three times. Finally, the protein was dissolved in buffer (8 M urea, $100 \mathrm{mM}$ TEAB, pH 8.0), and the protein concentration in the supernatant was estimated with a 2-D Quant kit (GE Healthcare, USA) according to the manufacturer's instructions. To ensure adequate coverage, three biological replicates of each group were collected.

\section{Proteomics and bioinformatics analysis}

After extraction, proteins were digested into peptides. TMT labeling, HPLC fractionation and LC-MS/MS were then used to analyze and quantify the dynamic changes of the proteome. To ensure adequate coverage, three biological replicates (i.e., six samples) were collected. The MS/MS data were processed using the Mascot search engine (v.2.3.0). Tandem mass spectra were searched against the SwissProt Green Plant database. For protein quantification, the MASCOT software package in NCBI were used in the present work. The Kyoto Encyclopedia of Genes and Genomes (KEGG) database was used to annotate the protein pathway. The protein-protein interaction network was obtained from the String database and the interactions between proteins were performed using Cytoscape software (3.4.0).

\section{TMT/iTRAQ Labeling (optional)}

After trypsin digestion, peptide was desalted by Strata X C18 SPE column (Phenomenex) and vacuumdried. Peptide was reconstituted in 0.5 M TEAB and processed according to the manufacturer's protocol for TMT kit/iTRAQ kit. Briefly, one unit of TMT/iTRAQ reagent were thawed and reconstituted in acetonitrile. The peptide mixtures were then incubated for $2 \mathrm{~h}$ at room temperature and pooled, desalted and dried by vacuum centrifugation.

\section{LC-MS/MS Analysis and Database Search}

The tryptic peptides were dissolved in $0.1 \%$ formic acid (solvent $A$ ), directly loaded onto a home-made reversed-phase analytical column (15-cm length, $75 \mu \mathrm{m}$ i.d.). The gradient was comprised of an increase from $6-23 \%$ solvent B ( $0.1 \%$ formic acid in $98 \%$ acetonitrile) over $26 \mathrm{~min}, 23-35 \%$ in 8 min and climbing to $80 \%$ in $3 \mathrm{~min}$ then holding at $80 \%$ for the last $3 \mathrm{~min}$, all at a constant flow rate of $400 \mathrm{~nL} / \mathrm{min}$ on an EASY-nLC 1000 UPLC system.

The peptides were subjected to NSI source followed by tandem mass spectrometry (MS/MS) in Q ExactiveTM Plus (Thermo) coupled online to the UPLC. The electrospray voltage applied was $2.0 \mathrm{kV}$. The $\mathrm{m} / \mathrm{z}$ scan range was 350 to 1800 for full scan, and intact peptides were detected in the Orbitrap at a resolution of 70,000. Peptides were then selected for MS/MS using NCE setting as 28 and the fragments were detected in the Orbitrap at a resolution of 17,500. A data-dependent procedure that alternated between one MS scan followed by $20 \mathrm{MS} / \mathrm{MS}$ scans with 15.0 s dynamic exclusion. Automatic gain control (AGC) was set at 5E4. Fixed first mass was set as $100 \mathrm{~m} / \mathrm{z}$.

The resulting MS/MS data were processed using Maxquant search engine (v.1.5.2.8). Tandem mass spectra were searched against human uniprot database concatenated with reverse decoy database. Trypsin/P was specified as cleavage enzyme allowing up to 4 missing cleavages. The mass tolerance for precursor ions was set as 20 ppm in First search and 5 ppm in Main search, and the mass tolerance for 
fragment ions was set as 0.02 Da. Carbamidomethyl on Cys was specified as fixed modification and Acetylation modification and oxidation on Met were specified as variable modifications. FDR was adjusted to $<1 \%$ and minimum score for modified peptides was set $>40$.

\section{Protein Functional Enrichment}

Proteins without detailed annotation were annotated by searching against the NCBI non-redundant protein database2 using PSI and PHI-BLAST programs3. Protein functional classification was performed on the basis of combination of information from KEGG pathway database4, UniProt database5, and the Gene Ontology protein database6, as well as literature.

\section{Enrichment of Gene Ontology analysis}

Proteins were classified by GO annotation into three categories: biological process, cellular compartment and molecular function. For each category, a two-tailed Fisher's exact test was employed to test the enrichment of the differentially modified protein against all identified proteins. The GO with a corrected pvalue $<0.05$ is considered significant.

\section{Enrichment of pathway analysis}

Encyclopedia of Genes and Genomes (KEGG) database was used to identify enriched pathways by a twotailed Fisher's exact test to test the enrichment of the differentially modified protein against all identified proteins. The pathway with a corrected $p$-value $<0.05$ was considered significant. These pathways were classified into hierarchical categories according to the KEGG website.

\section{Enrichment of protein domain analysis}

For each category proteins, InterPro (a resource that provides functional analysis of protein sequences by classifying them into families and predicting the presence of domains and important sites) database was researched and a two-tailed Fisher's exact test was employed to test the enrichment of the differentially modified protein against all identified proteins. Protein domains with a corrected $p$-value $<0.05$ were considered significant.

\section{Phosphoprotein Homology Modeling}

Three-dimensional structural models for phosphoproteins were generated using SWISS-MODEL comparative protein modeling server8(Biasini et al., 2014). Structures were visualized and analyzed using the Swiss-PdbViewer software (version 3.7). Functional domains were predicted by InterPro: the integrative protein signature database9.

\section{Abbreviations}

ALDH

NAD $(P)+$-dependent aldehyde dehydrogenases; CaM-BP:CaM-binding protein; CaM:calcium;CAT:Catalase; COG:Clusters of Orthologous Groups of proteins; MAP3K epsilon protein kinase 1; NDPK2, nucleoside 
diphosphate kinase 2 protein kinase OEC $\mathrm{PSIl}$ oxygen evolving complex; POD:peroxidase; SOD:Superoxide dismutase; TMT labeling:Tandem mass tags labeling; STP:serine/threonine-protein kinase;

\section{Declarations}

\section{Acknowledgements}

Not applicable.

\section{Funding}

This work was mainly supported by the National Natural Science Foundation of China (31070224) and the Science and Technology Department of Jilin Province (20130206059NY).

\section{Availability of data and materials}

The datasets generated during and/or analyzed during the current study are

available from the corresponding author on reasonable request.

\section{Authors' contributions}

$\mathrm{XZ}$ and $\mathrm{HX}$ designed the research; $\mathrm{YL}$ and $\mathrm{ZZ}$ prepared the plant materials for sequencing. $\mathrm{YL}$ carried out bioinformatics analysis of data; LY, HF and YT performed the experiments and statistical analyses; $Y L$ and $\mathrm{ZZ}$ collected data and researched literature, $\mathrm{YL}$ interpreted the data and wrote the manuscript. All authors read and approved the final manuscript.

\section{Competing interests}

The authors declare that they have no competing interests.

\section{Consent for publication}

Not applicable.

\section{Ethics approval and consent to participate}

Not applicable.

\section{References}

1. Thakur P, Kumar S, Malik JA, Berger JD, Nayyar H. Cold stress effects on reproductive development in grain crops: An overview. Environ Exp Bot. 2010;67:429-43. doi:10.1016/j.envexpbot.2009.09.004. 
2. Orvar BL, Sangwan V, Omann F, Dhindsa RS. Early steps in cold sensing by plant cells: the role of actin cytoskeleton and membrane fluidity. Plant J. 2000;23:785-94. doi:10.1046/j.1365313x.2000.00845.x.

3. Ding Y, Shi Y, Yang S. Advances and challenges in uncovering cold tolerance regulatory mechanisms in plants. New Phytol. 2019;222:1690-704. doi:10.1111/nph.15696.

4. Liu H, Wang F-F, Peng X-J, Huang J-H, Shen S-H. Global Phosphoproteomic Analysis Reveals the Defense and Response Mechanisms of Jatropha Curcas Seedling under Chilling Stress. Int J Mol Sci. 2019. doi:10.3390/ijms20010208.

5. Huang W, Zhang S-B, Cao K-F. The different effects of chilling stress under moderate light intensity on photosystem II compared with photosystem I and subsequent recovery in tropical tree species. Photosynth Res. 2010;103:175-82. doi:10.1007/s11120-010-9539-7.

6. Janmohammadi M, Zolla L, Rinalducci S. Low temperature tolerance in plants: Changes at the protein level. Phytochemistry. 2015;117:76-89. doi:10.1016/j.phytochem.2015.06.003.

7. Yuan P, Yang T, Poovaiah BW. Calcium Signaling-Mediated Plant Response to Cold Stress. IJMS. 2018;19:3896. doi:10.3390/ijms19123896.

8. Mittler R, Vanderauwera S, Gollery M, van Breusegem F. Reactive oxygen gene network of plants. Trends Plant Sci. 2004;9:490-8. doi:10.1016/j.tplants.2004.08.009.

9. Kamal MM, Ishikawa S, Takahashi F, Suzuki K, Kamo M, Umezawa T, et al. Large-Scale Phosphoproteomic Study of Arabidopsis Membrane Proteins Reveals Early Signaling Events in Response to Cold. Int J Mol Sci. 2020. doi:10.3390/ijms21228631.

10. Rampitsch C, Bykova NV. The beginnings of crop phosphoproteomics: exploring early warning systems of stress. Front Plant Sci. 2012;3:144. doi:10.3389/fpls.2012.00144.

11. Hsu C-C, Zhu Y, Arrington JV, Paez JS, Wang P, Zhu P, et al. Universal Plant Phosphoproteomics Workflow and Its Application to Tomato Signaling in Response to Cold Stress. Mol Cell Proteomics. 2018;17:2068-80. doi:10.1074/mcp.TIR118.000702.

12. Pi Z, Zhao M-L, Peng X-J, Shen S-H. Phosphoproteomic Analysis of Paper Mulberry Reveals Phosphorylation Functions in Chilling Tolerance. J Proteome Res. 2017;16:1944-61. doi:10.1021/acs.jproteome.6b01016.

13. Lu Z-S, Chen Q-S, Zheng Q-X, Shen J-J, Luo Z-P, Fan K, et al. Proteomic and Phosphoproteomic Analysis in Tobacco Mosaic Virus-Infected Tobacco (Nicotiana tabacum). Biomolecules 2019. doi:10.3390/biom9020039.

14. Zhou X, Chen S, Wu H, Yang Y, Xu H. Biochemical and proteomics analyses of antioxidant enzymes reveal the potential stress tolerance in Rhododendron chrysanthum Pall. Biol Direct. 2017;12:10. doi:10.1186/s13062-017-0181-6.

15. Singh M. Turnover of D1 Protein Encoded by psbA Gene in Higher Plants and Cyanobacteria Sustains Photosynthetic Efficiency to Maintain Plant Productivity Under Photoinhibitory Irradiance. Photosynthetica. 2000;38:161-9. doi:10.1023/A:1007297227403. 
16. Salonen M, Aro E-M, Rintamäki E. Reversible phosphorylation and turnover of the D1 protein under various redox states of Photosystem II induced by low temperature photoinhibition. Photosynth Res. 1998;58:143-51. doi:10.1023/A:1006155223221.

17. Koivuniemi A, Aro EM, Andersson B. Degradation of the D1- and D2-proteins of photosystem II in higher plants is regulated by reversible phosphorylation. Biochemistry. 1995;34:16022-9. doi:10.1021/bi00049a016.

18. Grebe S, Trotta A, Bajwa AA, Mancini I, Bag P, Jansson S, et al. Specific thylakoid protein phosphorylations are prerequisites for overwintering of Norway spruce (Picea abies) photosynthesis. Proc Natl Acad Sci U S A. 2020;117:17499-509. doi:10.1073/pnas.2004165117.

19. Merry R, Jerrard J, Frebault J, Verhoeven A. A comparison of pine and spruce in recovery from winter stress; changes in recovery kinetics, and the abundance and phosphorylation status of photosynthetic proteins during winter. Tree Physiol. 2017;37:1239-50. doi:10.1093/treephys/tpx065.

20. Allahverdiyeva Y, Suorsa M, Rossi F, Pavesi A, Kater MM, Antonacci A, et al. Arabidopsis plants lacking PsbQ and PsbR subunits of the oxygen-evolving complex show altered PSIl super-complex organization and short-term adaptive mechanisms. Plant J. 2013;75:671-84. doi:10.1111/tpj.12230.

21. Tola AJ, Jaballi A, Germain H, Missihoun TD. Recent Development on Plant Aldehyde Dehydrogenase Enzymes and Their Functions in Plant Development and Stress Signaling. Genes (Basel). 2020. doi:10.3390/genes12010051.

22. Sparla F, Fermani S, Falini G, Zaffagnini M, Ripamonti A, Sabatino P, et al. Coenzyme site-directed mutants of photosynthetic A4-GAPDH show selectively reduced NADPH-dependent catalysis, similar to regulatory AB-GAPDH inhibited by oxidized thioredoxin. J Mol Biol. 2004;340:1025-37. doi:10.1016/j.jmb.2004.06.005.

23. Hsu CH, Hsu YT. Biochemical responses of rice roots to cold stress. Bot Stud. 2019;60:14. doi:10.1186/s40529-019-0262-1.

24. Qi W, Wang F, Ma L, Qi Z, Liu S, Chen C, et al. Physiological and Biochemical Mechanisms and Cytology of Cold Tolerance in Brassica napus. Front Plant Sci. 2020;11:1241. doi:10.3389/fpls.2020.01241.

25. Mittler R, Vanderauwera S, Gollery M, van Breusegem F. Reactive oxygen gene network of plants. Trends Plant Sci. 2004;9:490-8. doi:10.1016/j.tplants.2004.08.009.

26. Mhamdi A, Queval G, Chaouch S, Vanderauwera S, van Breusegem F, Noctor G. Catalase function in plants: a focus on Arabidopsis mutants as stress-mimic models. J Exp Bot. 2010;61:4197-220. doi:10.1093/jxb/erq282.

27. Chang T-S, Jeong W, Choi SY, Yu S, Kang SW, Rhee SG. Regulation of peroxiredoxin I activity by Cdc2mediated phosphorylation. J Biol Chem. 2002;277:25370-6. doi:10.1074/jbc.M110432200.

28. Han F, Chen H, Li X-J, Yang M-F, Liu G-S, Shen S-H. A comparative proteomic analysis of rice seedlings under various high-temperature stresses. Biochim Biophys Acta. 2009;1794:1625-34. doi:10.1016/j.bbapap.2009.07.013. 
29. Moon H, Lee B, Choi G, Shin D, Prasad DT, Lee O, et al. NDP kinase 2 interacts with two oxidative stress-activated MAPKs to regulate cellular redox state and enhances multiple stress tolerance in transgenic plants. Proc Natl Acad Sci U S A. 2003;100:358-63. doi:10.1073/pnas.252641899.

30. Andrews C, Xu Y, Kirberger M, Yang JJ. Structural Aspects and Prediction of Calmodulin-Binding Proteins. Int J Mol Sci. 2020. doi:10.3390/ijms22010308.

31. Reddy ASN, Ali GS, Celesnik H, Day IS. Coping with stresses: roles of calcium- and calcium/calmodulin-regulated gene expression. Plant Cell. 2011;23:2010-32. doi:10.1105/tpc.111.084988.

32. Garg G, Yadav S, Ruchi, Yadav G. Key Roles of Calreticulin and Calnexin Proteins in Plant Perception under Stress Conditions: A Review. Advances in Life Sciences. 2015;5:18-26.

33. Takemiya A, Sugiyama N, Fujimoto H, Tsutsumi T, Yamauchi S, Hiyama A, et al. Phosphorylation of BLUS1 kinase by phototropins is a primary step in stomatal opening. Nat Commun. 2013;4:2094. doi:10.1038/ncomms3094.

\section{Figures}



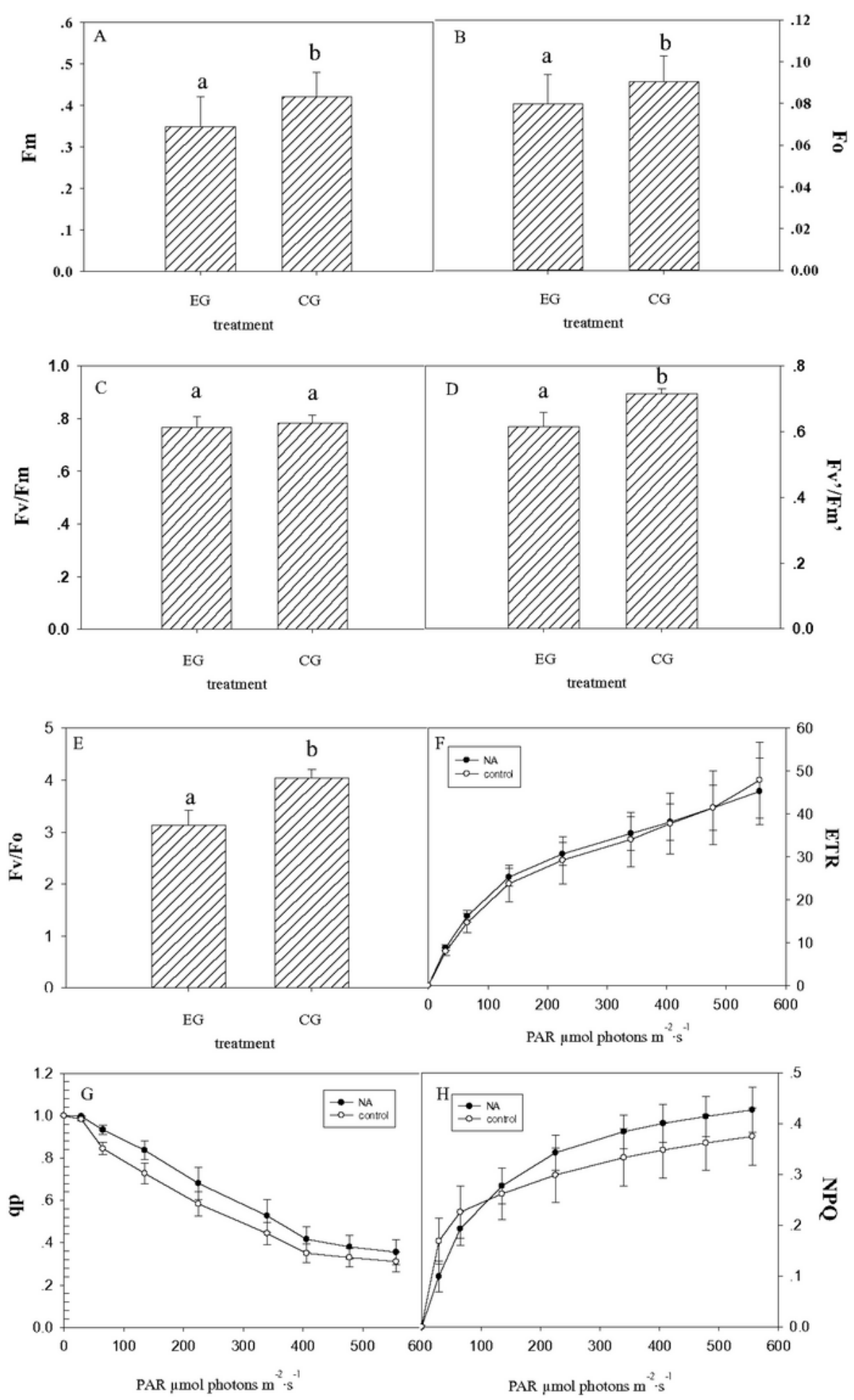

\section{Figure 1}

Photosynthetic characteristics in R. chrysanthum under cold stress. Lower case letters indicate significant differences among different treatments $(p<0.05)$. (A) initial minimum fluorescence (Fo); (B) maximal fluorescence (Fm); (C) Maximum quantum yield of PSII (Fv/Fm); (D) effective quantum yield of PSII $\left(F^{\prime} / F^{\prime}\right) ;(E)$ Potential Activity of Optical System II (Fv/Fo); (F) relative electron transport rate (ETR); (G) 
Mean nonphotochemical quenching (NPQ) as a function of PAR; $(\mathrm{H})$ Mean photochemical quenching (qP) as a function of PAR.

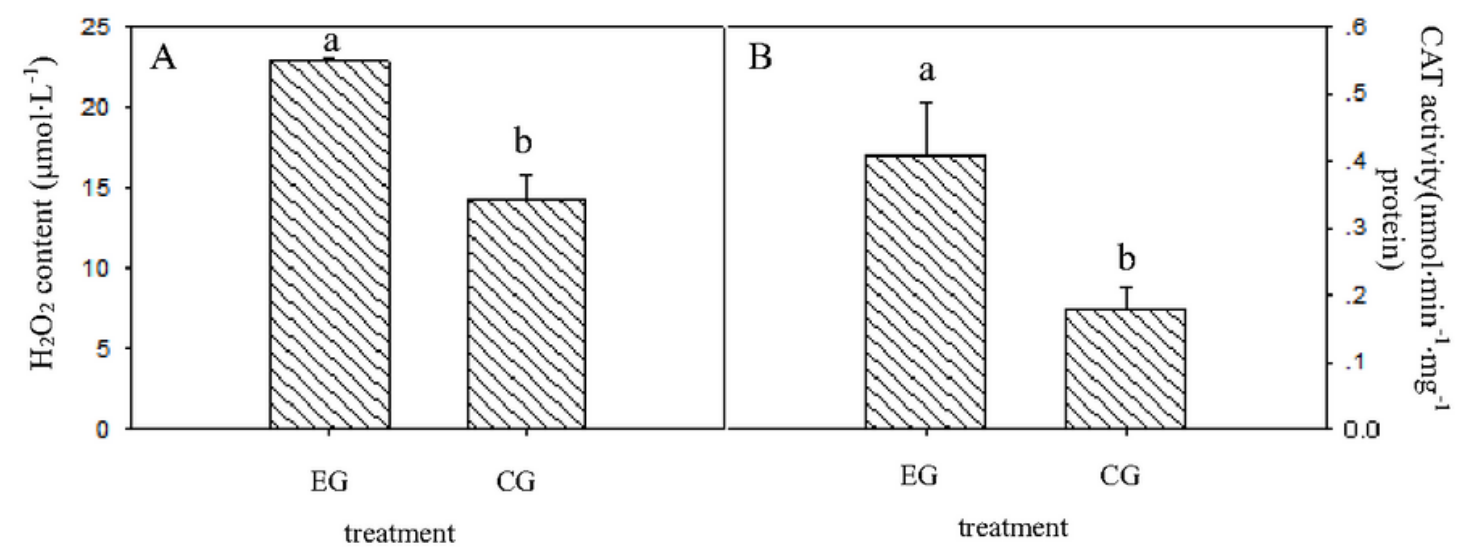

Figure 2

$\mathrm{H} 2 \mathrm{O} 2$ content and catalase activities in rhododendron aureum under cold stress. (A) H2O2 content; (B) catalase (CAT) activities 

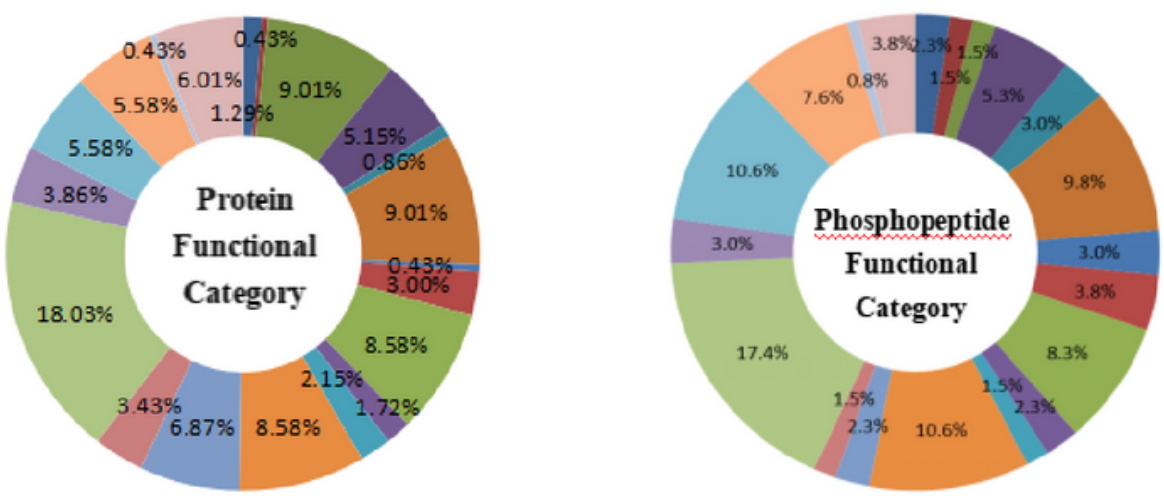

- RNA processing and modification

Energy production and conversion

- Nucleotide transport and metabolism

- Coenzyme transport and metabolism

- Translation, ribosomal structure and biogenesis

- Cell wall/membrane/envelope biogenesis

Inorganic ion transport and metabolism

1] General function prediction only

II Signal transduction mechanisms

Nuclear structure

\begin{abstract}
Chromatin structure and dynamics
- Amino acid transport and metabolism

Carbohydrate transport and metabolism

Lipid transport and metabolism

- Transcription

[1 Posttranslational modification, protein turnover, chaperones

- Secondary metabolites biosynthesis, transport and catabolism

E Function unknown

Intracellular trafficking, secretion, and vesicular transport

in cytoskeleton
\end{abstract}

B
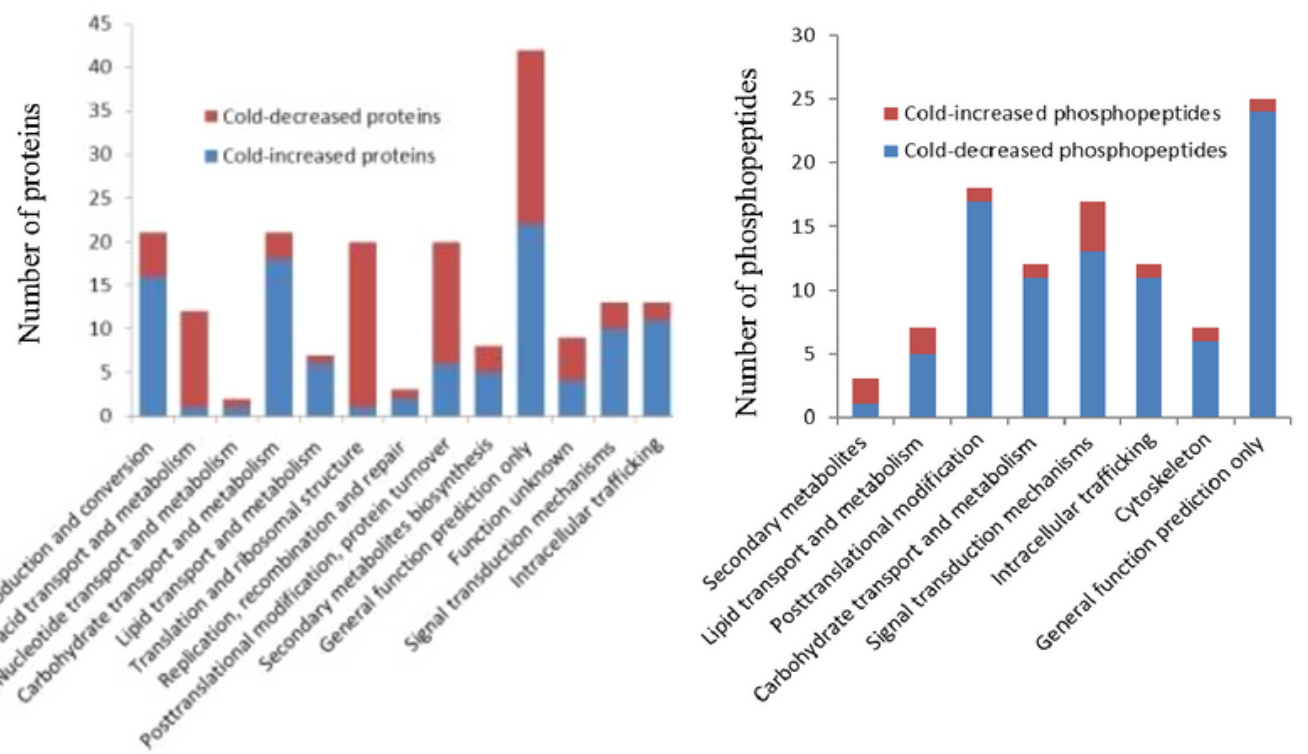

\title{
Figure 3
}

Functional categorization and abundance patterns of cold stress-responsive proteins and phosphopeptides in rhododendron aureum leaves. (A) A total of 350 cold stress-responsive proteins were classified into 20 functional categories, and a total of 274 cold stress-responsive phosphopeptides were classified into 20 functional categories. The percentage of proteins in each functional category is shown 
in the pie; (B) Abundance patterns of cold stress-responsive proteins and phosphopeptides in each functional category.

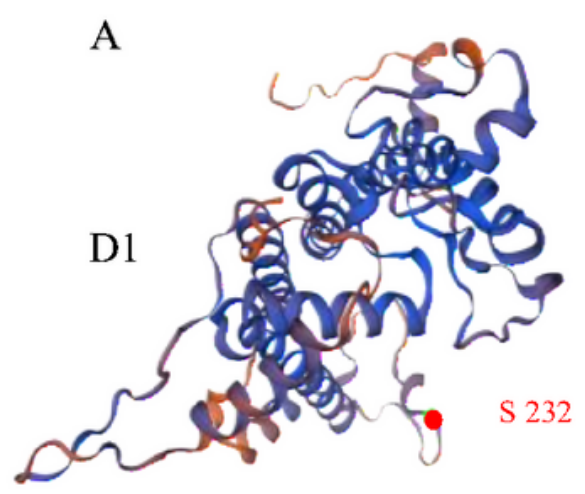

B
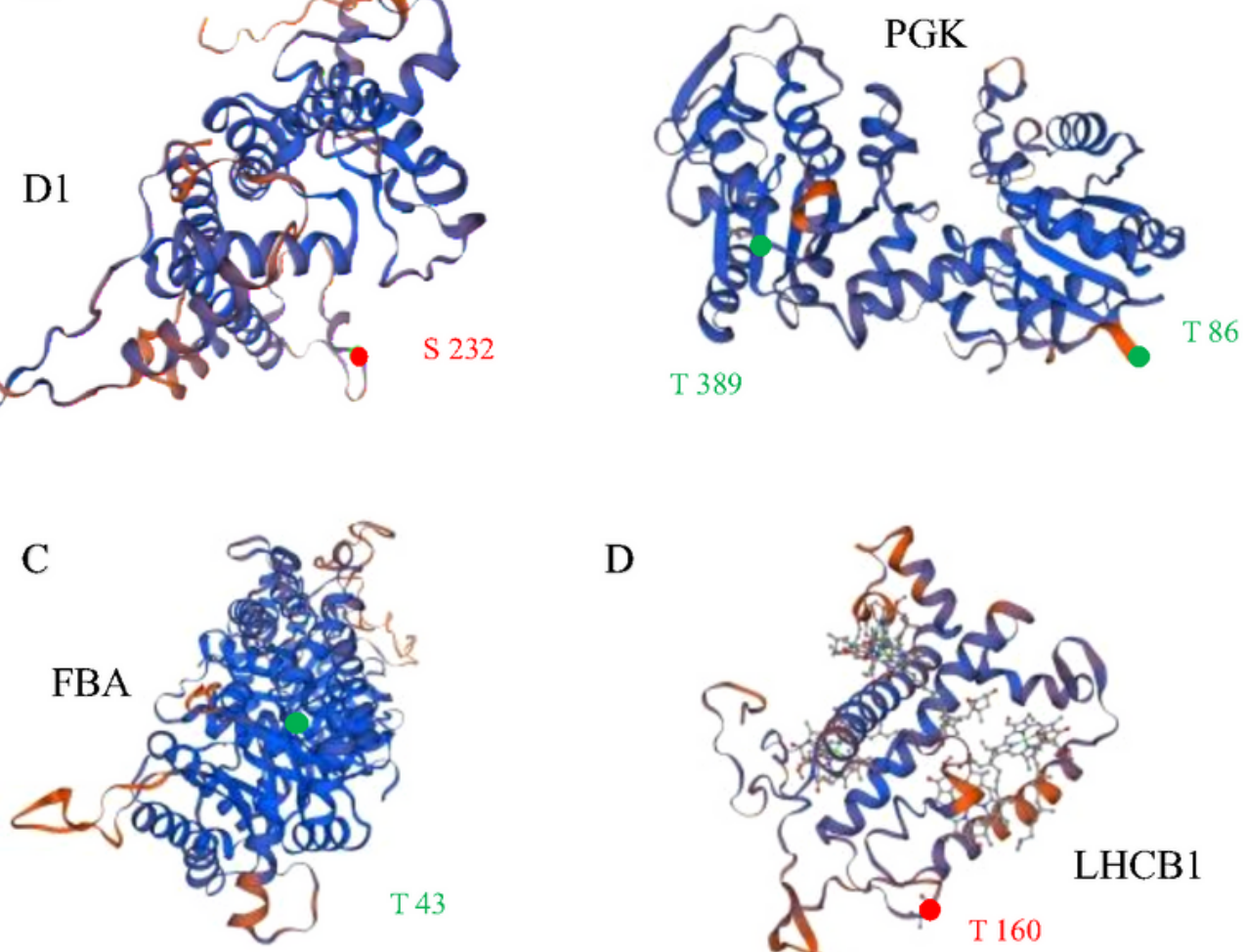

$\mathrm{D}$

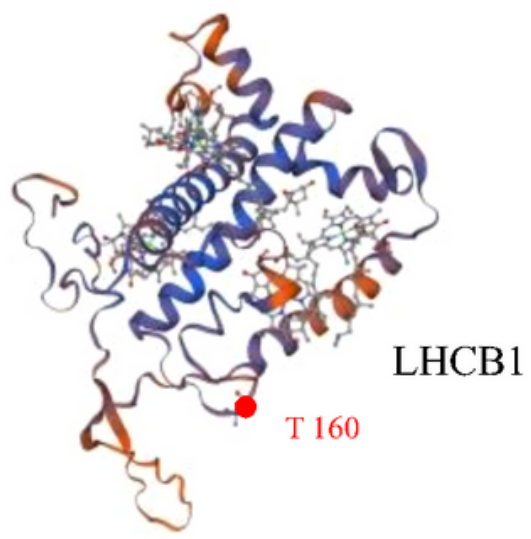

E

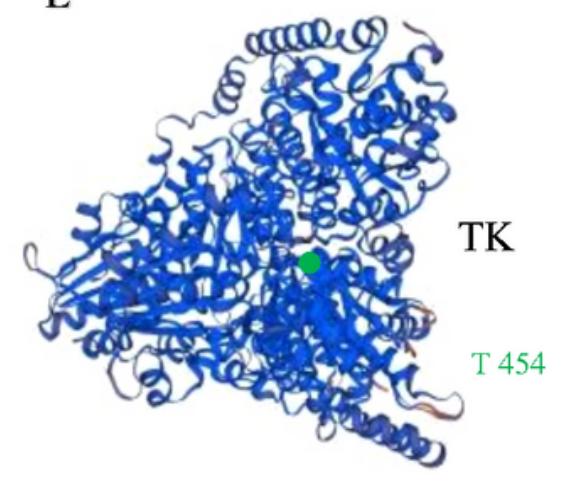

$\mathrm{F}$

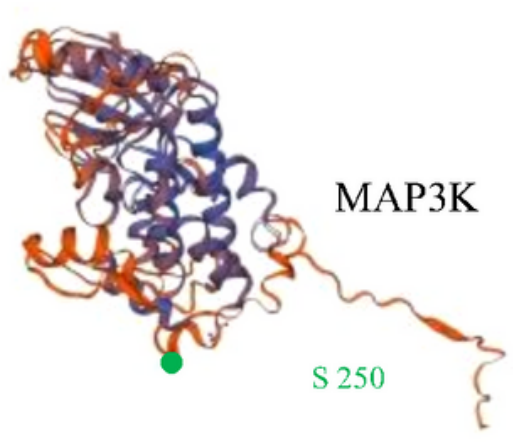

\section{Figure 4}

Homology models of the heat stress-responsive phosphoproteins from spinach leaves in three dimensions. (A) Photosystem II protein D1; (B) phosphoglycerate kinase (PGK); (C) fructose-bisphosphate aldolase (FBA); (D) light-harvesting complex II chlorophyll a/b binding protein 1(LHCB1); (E) transketolase 
(TK); (F) MAP3K epsilon protein kinase 1 OS=Arabidopsis thaliana (MAP3K). The phosphorylation sites are shown with balls in red (increased phosphorylation level) and green (decreased phosphorylation level). Domains from the phosphoprotein and corresponding names are highlighted in the same color.

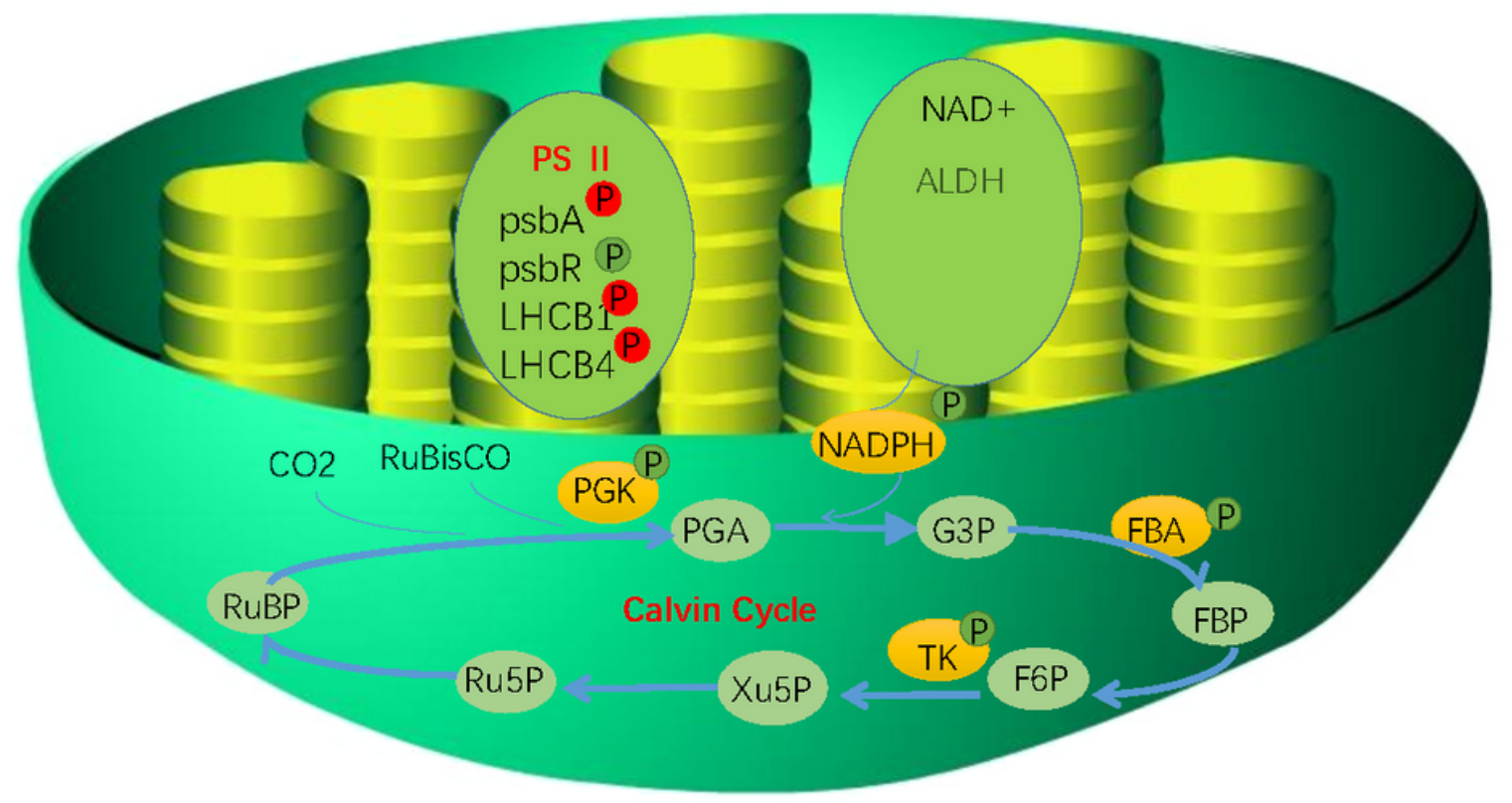

\section{Figure 5}

Schematic representation of cold stress-responsive photosynthesis in rhododendron chrysanthum leaves. Cold stress-responsive signal transduction, ROS homeostasis, and transcriptional regulation in rhododendron chrysanthum. Protein names in red and green represent increased and decreased protein abundances under cold stress, respectively. Substrate names with red and green indicate the coldincreased and cold-decreased contents, respectively. Arrows with solid and dashed lines represent direct stimulation/single-step reaction and indirect stimulation/multi-step reaction, respectively. A " $P$ " in red and green circles indicates increased and decreased phosphorylation levels of the corresponding proteins, respectively. $A$ " $P$ " in green circles indicates decreased phosphorylation levels of the corresponding proteins. 3PGA, 3-phosphoglycerate; F6P, fructose 6-phosphate; FBA, Fructose-bisphosphate aldolase; FBP, fructose-1,6-bisphosphate; G3P, glyceraldehyde 3-phosphate; NDH, NAD(P)H dehydrogenase; PS, photosystem; Ru5P, ribulose-5-phosphate; RuBisCO, ribulose bisphosphate carboxylase/ oxygenase; RuBP, ribulose-1,5-bisphosphate; TK, transketolase; Xu5P, ketose xylulose-5-phosphate. 


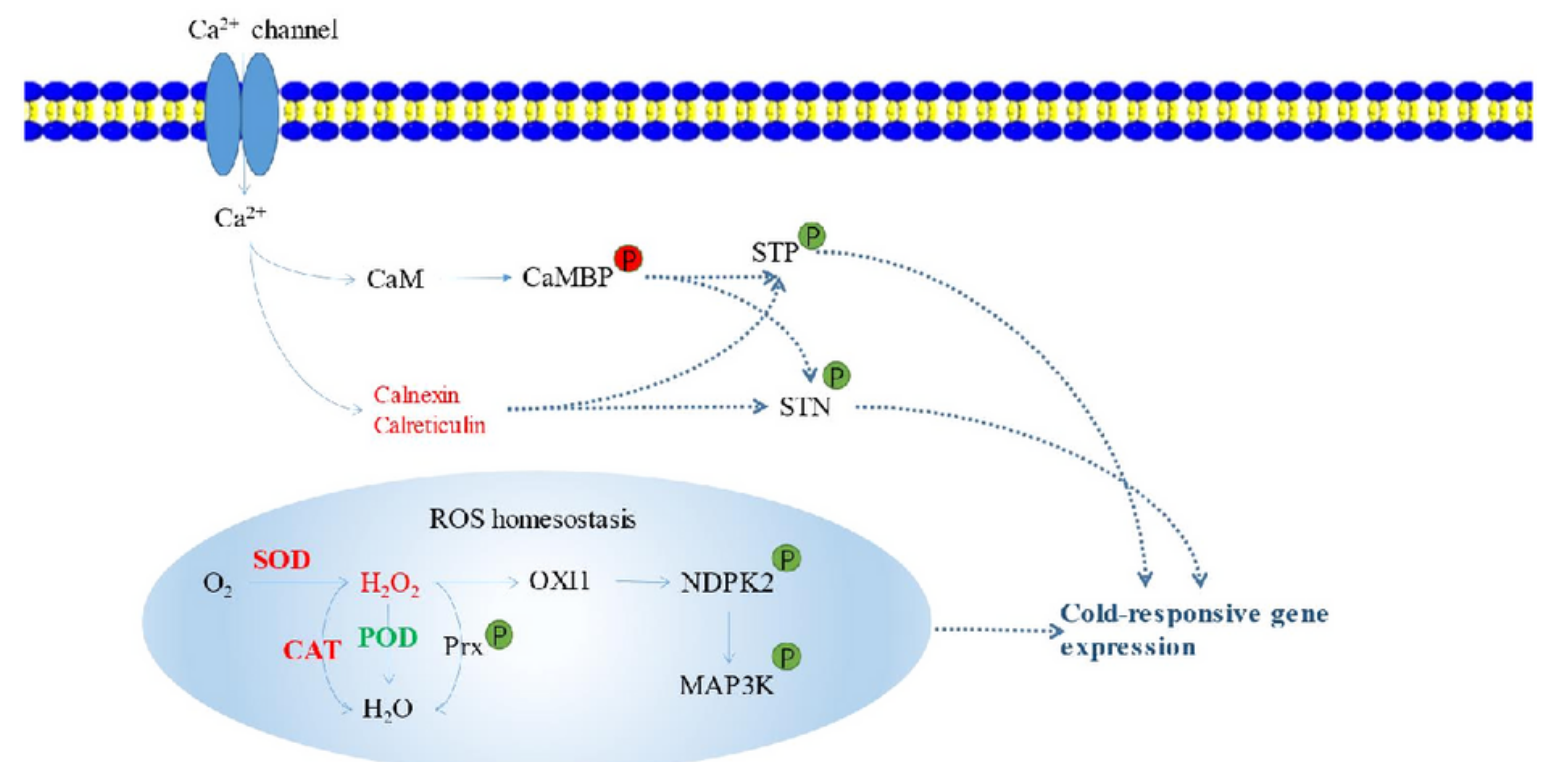

Figure 6

Cold stress-responsive signal transduction in rhododendron chrysanthum. Protein names in red and green represent increased and decreased protein abundances under cold stress, respectively. Substrate names with red and green indicate the cold-increased and cold-decreased contents, respectively. Arrows with solid and dashed lines represent direct stimulation/single-step reaction and indirect stimulation/multistep reaction, respectively. The " $\mathrm{T}$ " shape line represents inhibition. A " $\mathrm{P}$ " in red and green circles indicates increased and decreased phosphorylation levels of the corresponding proteins, respectively; CaM, calmodulin; CaMBP, calmodulin-binding protein; CAT, catalase; MAP3K epsilon protein kinase 1; NDPK2, nucleoside diphosphate kinase 2 protein kinase; POD, peroxidase; Prx, peroxiredoxin; ROS, reactive oxygen species; SOD, superoxide dismutase; STK, serine/threonine-protein kinase; STP, serine/threonineprotein phosphorylase; 